\title{
The influence of the axial rotation on the degenerate dwarfs characteristics
}

\author{
Vavrukh M., Smerechynskyi S., Dzikovskyi D. \\ Ivan Franko National University of Lviv \\ 8 Kyrylo and Methodiy Str., 79005 Lviv, Ukraine
}

(Received 26 June 2017)

\begin{abstract}
The influence of the axial rotation on degenerate dwarfs characteristics is calculated within the three- and four parametric model. It was shown that the relative increase of dwarf's mass is about $5 \%$. Therefore the maximal mass in standard model with paramagnetic electron subsystem reaches $1.52 M_{\odot}$, and in the model with spin-polarized electron subsystem is $2.15 M_{\odot}$. The dependence of the dwarf's shape was found as a function of the model parameters (the density in the stellar center, the chemical composition parameter, the rotation frequency and the degree of polarization).
\end{abstract}

Keywords: degenerate dwarf, the density in the stellar center, the chemical composition parameter, the rotation frequency, the degree of polarization.

2000 MSC: $85 \mathrm{~A} 15,83 \mathrm{C} 25,82 \mathrm{D} 35$

UDC: $524.31 .084,524.384,524.352 .3$

\section{Introduction}

Degenerate dwarfs are the simplest type of compact objects, which formed at the final stage of the evolution of not massive stars. The first dwarf - the faint companion of brightest star in the Northern sky of Sirius - was discovered by american astronomer U. Adams in 1914 [1]. It is believed now that the degenerate dwarfs are the most common types of stars in the universe and there were registered several thousand of dwarfs with different characteristics in the solar vicinity. Their masses do not exceed $1.5 M_{\odot}$, radii are in the range $(1 \div 3) \cdot 10^{-2} R_{\odot}$, and the luminosity is varying between $10^{-5} L_{\odot}$ and $10 L_{\odot}$, where $M_{\odot}, R_{\odot}, L_{\odot}$ are the characteristics of the Sun.

The theory of cold dwarfs was created in the first half of the last century in the works of A. Fowler [2] and S. Chandrasekhar [3,4]. It is based on the idea, that without any energy sources the stability of degenerate dwarfs is provided by the pressure of degenerate relativistic electron gas at high concentrations [5]. At the densities of the order $10^{5} \mathrm{~g} / \mathrm{cm}^{3}$ the matter of dwarfs is in the metallic state in extreme conditions of high pressure and temperature, when there is no localized states of electrons and the electron subsystem is relativistic completely degenerate and poorly ideal.

Within two-component model, that consists of degenerate relativistic electron gas at $T=0 \mathrm{~K}$ in the paramagnetic state and nuclear subsystem, which is described as continuous classic environment, S. Chandrasekhar found the existence of the upper limit of the dwarf mass and peculiar relation between mass and radius. According to the last one all dwarfs on the plane mass-radius must be on the same curve. In the last century the S. Chandrasekhar's theory was generalized by consideration of cooling of dwarfs [6], the influence of particle interactions [5], neutronization process [7], stability of dwarfs [8] and et. al. Development of theory was constrained by the lack of observational data.

The situation has been changed at the end of the last century with discovering of dwarfs with a large diversity of characteristics using modern space observatories. That dwarfs cannot be interpreted in the frame of S. Chandrasekhar's model. It is the complicated task to create a correct general theory of the structure of degenerate dwarf, which also would take into account other important factors 
- incomplete degeneration of electrons subsystem, variable along the radius chemical composition, particle interactions, axial rotation, influence of the magnetic fields and general relativity effects. According to the hypothesis, that the massive dwarfs can be precursors of type Ia supernova, urgent necessity is to find the mechanisms, which contribute to increase of the degenerate dwarfs' mass. It was shown in our works, that incomplete degeneration increases the dwarfs mass, but for massive stars, which are on the stability edge, it is insignificant $[9,10]$. Particle interactions decrease the internal pressure and it leads to decreasing mass [11]. Increase of mass of single dwarfs can be caused by the magnetic fields and axial rotation. In work [12] it was found that the spin-polarization of electron subsystem of dwarf, caused by the influence of a magnetic field, increases its mass, and in the case of full polarization the maximal mass exceeds the S. Chandrasehar's limit by $\sqrt{2}$ times. For the dwarfs in the double systems there can be additional mechanism - accretion, which may be the most important.

In this work we consider the influence of axial rotation of dwarf on its characteristics, which can be important as for single stars, as well as dwarfs in binary systems.

\section{The equilibrium equation of dwarf with axial rotation}

To investigate the influence of rotation on dwarf's characteristics, we have used two-component S. Chandrasekhar's model with ideal relativistic electron subsystem in paramagnetic phase and solid body rotation is considered. The structure of star is determined by the equation of mechanical equilibrium

$$
\nabla P(\mathbf{r})=-\rho(\mathbf{r}) \nabla\left\{\Phi_{\text {grav }}(\mathbf{r})+\Phi_{c}(\mathbf{r})\right\}
$$

where

$$
P(\mathbf{r})=8 \frac{\pi m_{0}^{4} c^{5}}{3 h^{3}} \int_{0}^{x(\mathbf{r})} \frac{t^{4} d t}{\sqrt{1+t^{2}}}
$$

is a local value of pressure of the degenerate relativistic electron gas at $T=0 K$,

$$
x(\mathbf{r})=\frac{\hbar}{m_{0} c}\left(3 \pi^{2} n(\mathbf{r})\right)^{1 / 3}
$$

is a local value of the relativistic parameter in a point with the number density of electrons $n(\mathbf{r}), m_{0}$ is the electron mass;

$$
\rho(\mathbf{r})=m_{u} \mu_{e} n(\mathbf{r})=\frac{m_{u} \mu_{e}}{3 \pi^{2}} x^{3}(\mathbf{r})\left(\frac{m_{0} c}{\hbar}\right)^{3}
$$

is a density of matter concentrated in the nuclei, $m_{u}$ is the atomic mass unit, $\mu_{e}=\langle A / z\rangle$ is the chemical composition parameter, which is considered a constant ( $A$ is the mass number of the nucleus, $z$ is its charge);

$$
\Phi_{\text {grav }}(\mathbf{r})=-G \int \frac{\rho\left(\mathbf{r}^{\prime}\right) d \mathbf{r}^{\prime}}{\left|\mathbf{r}-\mathbf{r}^{\prime}\right|}
$$

is the gravitational potential in the point with radius-vector $\mathbf{r}$, and the centrifugal potential

$$
\Phi_{c}(\mathbf{r})=-\frac{1}{2} \Omega^{2} r^{2} \sin ^{2} \theta .
$$

We use here the spherical coordinate system with the beginning in the stellar center and axis $O z$ coincides with the axis of rotation with the frequency $\Omega$.

Taking into account the formulae (2)-(6) the equilibrium equation can be rewritten in the following form:

$$
\Delta\left\{\left[1+x^{2}(\mathbf{r})\right]^{1 / 2}-1-\frac{\Omega^{2}}{2} \frac{m_{u} \mu_{e}}{m_{0} c^{2}} r^{2} \sin ^{2} \theta\right\}=-\frac{32 \pi^{2} G\left(m_{u} \mu_{e} m_{0} c^{2}\right)^{2}}{3(h c)^{3}} x^{3}(\mathbf{r}) .
$$


Introducing the dimensionless variables

$$
\xi=r / \lambda, \quad y(\xi, \theta)=\varepsilon_{0}^{-1}\left\{\left[1+x^{2}(\mathbf{r})\right]^{1 / 2}-1\right\}, \quad \varepsilon_{0}=\varepsilon_{0}\left(x_{0}\right)=\left[\left(1+x_{0}^{2}\right)^{1 / 2}-1\right]
$$

and auxiliary function

$$
\varphi(\xi, \theta)=y(\xi, \theta)-\omega(\xi, \theta), \quad \omega(\xi, \theta)=\tilde{\Omega}^{2} \xi^{2} \sin ^{2} \theta, \quad \tilde{\Omega}^{2} \equiv \frac{\Omega^{2} m_{u} \mu_{e} \lambda^{2}}{2 \varepsilon_{0} m_{0} c^{2}}
$$

the equation (7) is transformed to the equation for function $\varphi(\xi, \theta)$ :

$$
\Delta(\xi, \theta) \varphi(\xi, \theta)=-\left\{[\varphi(\xi, \theta)+\omega(\xi, \theta)]^{2}+\frac{2}{\varepsilon_{0}}[\varphi(\xi, \theta)+\omega(\xi, \theta)]\right\}^{3 / 2},
$$

where $\Delta(\xi, \theta)$ is the Laplace operator in dimensionless variables $(\xi, \theta)$, and the length scale $\lambda$ is determined by expression

$$
\frac{32 \pi^{2} G}{3(h c)^{3}}\left\{m_{0} c^{2} \varepsilon_{0} m_{u} \mu_{e} \lambda\right\}^{2}=1
$$

The equation (10) is the two-dimensional nonlinear differential equation of second order in partial variables. At fixed value of angle $\theta$ the equation becomes one-dimensional, and $\theta$ in it appears as independent parameter $(0 \leqslant \theta \leqslant \pi)$ :

$$
\frac{d^{2}}{d \xi^{2}} \varphi(\xi, \theta)+\frac{2}{\xi} \frac{d}{d \xi} \varphi(\xi, \theta)=-\left\{[\varphi(\xi, \theta)+\omega(\xi, \theta)]^{2}+\frac{2}{\varepsilon_{0}}[\varphi(\xi, \theta)+\omega(\xi, \theta)]\right\}^{3 / 2}
$$

Regular (at $\xi \rightarrow 0$ ) solutions of the equation correspond boundary conditions

$$
\varphi(0, \theta)=1, \quad \frac{d}{d \xi} \varphi(\xi, \theta)=0 \quad \text { by } \quad \xi \rightarrow 0 .
$$

In the equation (12) also appears dimensionless parameter $\tilde{\Omega}^{2}$, which is fairly large values of the relativistic parameter $x_{0}$ is a small value:

$$
\tilde{\Omega}=\Omega T_{0} \mu_{e}^{-1 / 2} \varepsilon_{0}^{-3 / 2},
$$

where

$$
\begin{aligned}
& T_{0}=\sqrt{2} M_{0} G\left(\frac{m_{u}}{m_{0}}\right)^{3 / 2} c^{-3} \\
& M_{0}=\left(\frac{3}{2}\right)^{1 / 2} \frac{1}{4 \pi}\left(\frac{h c}{G}\right)^{3 / 2} m_{u}^{-2}
\end{aligned}
$$

are the scales of rotation period and mass $\left(M_{0} \approx\right.$ $\left.2.89 \ldots M_{\odot}, T_{0} \approx 1.6 \ldots s\right)$ for degenerate dwarfs.

The equation solutions (12) are found by us

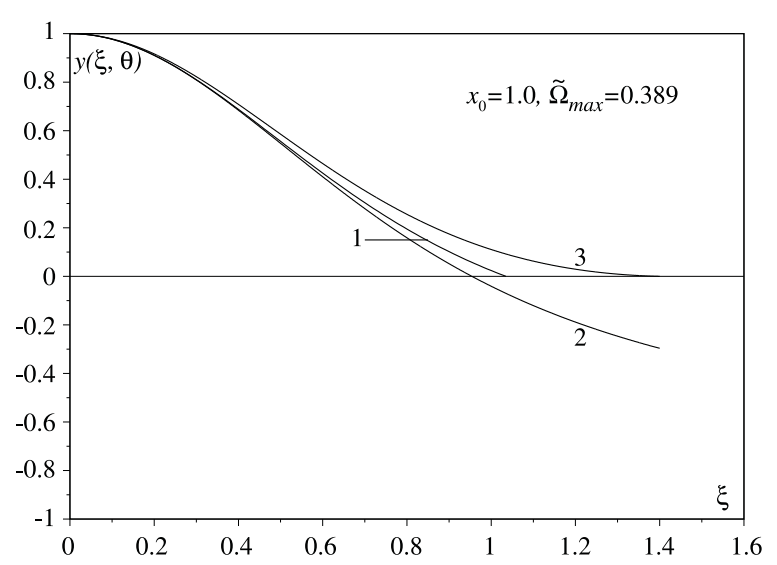

Fig. 1. The equation (12) solutions at fixed value of the relativistic parameter $x_{0}$ and the maximal rotational frequency $\tilde{\Omega}_{\max }$ (curve 1 corresponds to standard model, 2 - function $\varphi(\xi, \theta), 3$ - function $y(\xi, \theta))$. with numerical integration. In Fig. 1 was illustrated the solution at $\theta=\pi / 2$ : curve 1 corresponds to dwarf without rotation $(\tilde{\Omega}=0)$, and curves 2 and 3 represent the functions $\varphi(\xi, \theta)$ and $y(\xi, \theta)$ at the maximal possible rotational frequency, which are determined by conditions:

$$
y\left(\xi_{1}, \pi / 2\right)=0,\left.\quad \frac{\partial}{\partial \xi} y(\xi, \pi / 2)\right|_{\xi=\xi_{1}}=0,
$$


where $\xi_{1}$ is the dimensionless equatorial radius, which corresponds to the maximal rotational frequency. In Fig. $2 a$ was depicted dependence of the $\tilde{\Omega}_{\max }$, and in Fig. $2 b-\Omega_{\max }$ on the relativistic parameter $x_{0}$.
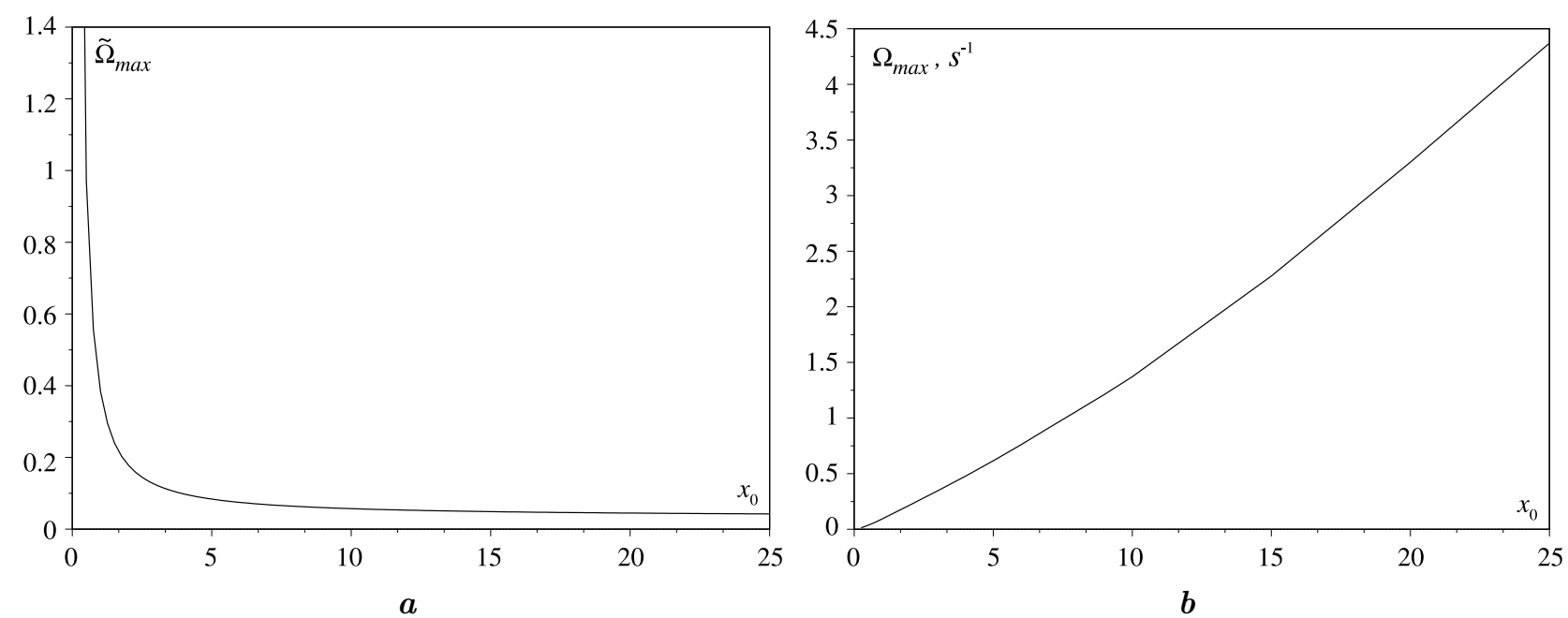

Fig. 2. Dependence of the $\tilde{\Omega}_{\max }$ and $\Omega_{\max }$ on the relativistic parameter $x_{0}$.

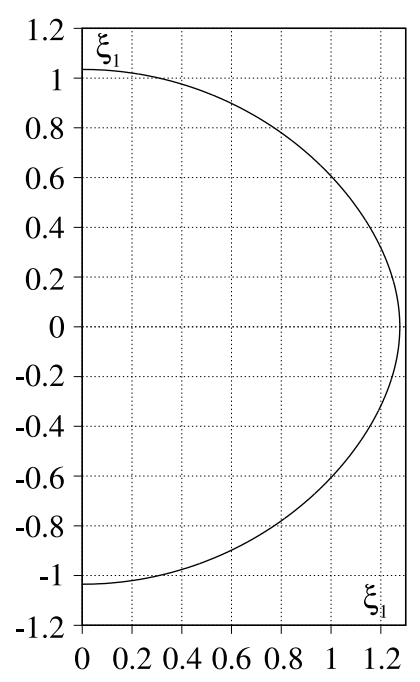

Fig. 3. Shape of the dwarf in the model with parameters $x_{0}=1.0, \Omega=0.95 \tilde{\Omega}_{\max }$.
The polar radius coincides with the radius of dwarf without rotation and determined by the condition

$$
\varphi_{0}\left(\xi_{1}^{0}\right)=0
$$

where $\varphi_{0}(\xi)$ is the equation solutions $(12)$ at $\tilde{\Omega}=0$.

The condition

$$
y\left(\xi_{1}, \theta\right)=0 \quad \text { by } \quad \tilde{\Omega}^{2}=\text { const }
$$

determines shape of surface at fixed value of the relativistic parameter $x_{0}$ (see Fig. 3). In dimensional variables, this surface is described by the expression

$$
R\left(x_{0}, \mu_{e} \mid \theta\right)=\frac{R_{0}}{\mu_{e} \varepsilon_{0}} \xi_{1}\left(\theta \mid x_{0}\right)
$$

where

$$
R_{0}=\left(\frac{3}{2}\right)^{1 / 2} \frac{1}{4 \pi}\left(\frac{h^{3}}{c G}\right)^{1 / 2} \frac{1}{m_{u} m_{0}},
$$

\section{The characteristics of dwarf with rotation}

We consider the model which has three parameters $\left(\mu_{e}, x_{0}\right.$ and $\left.\Omega\right)$. The formulae $(12)-(20)$ give the possibility to calculate dependence of the macroscopic characteristics of star on model parameter at arbitrary value $\mu_{e}$ and $x_{0}$ in the frequency range $0 \leqslant \Omega \leqslant \Omega_{\max }\left(x_{0}\right)$. Mass of a dwarf is

$$
\begin{aligned}
& M\left(x_{0}, \mu_{e}, \Omega\right)=\int \rho(\mathbf{r}) d \mathbf{r}=\frac{M_{0}}{\mu_{e}^{2}} \mathcal{M}\left(x_{0}, \tilde{\Omega}\right), \\
& \mathcal{M}\left(x_{0}, \tilde{\Omega}\right)=\int_{0}^{\pi / 2} \sin \theta d \theta \int_{0}^{\xi_{1}(\theta)} \xi^{2}\left\{y^{2}(\xi, \theta)+\frac{2}{\varepsilon_{0}} y(\xi, \theta)\right\}^{3 / 2} d \xi,
\end{aligned}
$$

Mathematical Modeling and Computing, Vol.4, No.1, pp.107-115 (2017) 
where $\xi_{1}(\theta)$ is determined by the condition (18). The dependence of maximal mass of dwarf, which corresponds to $\tilde{\Omega}_{\max }\left(x_{0}\right)$ on the relativistic parameter is shown in Fig. 4. It was also shown dependence on the parameter $x_{0}$ of dwarf mass without rotation. As can be seen from Fig. $M\left(x_{0}, \mu_{e}, \Omega\right)$ is monotonically increasing function of the relativistic parameter, similarly to the case $M\left(x_{0}, \mu_{e}\right)$ without rotation. Within large values of the relativistic parameter a mass of dwarf with axial rotation can exceed corresponding one without rotation by about $5 \%$. Thus the maximal mass of a dwarf can exceed the value of $1.52 M_{\odot}$. Dwarfs with such masses are found in the binary systems [6].

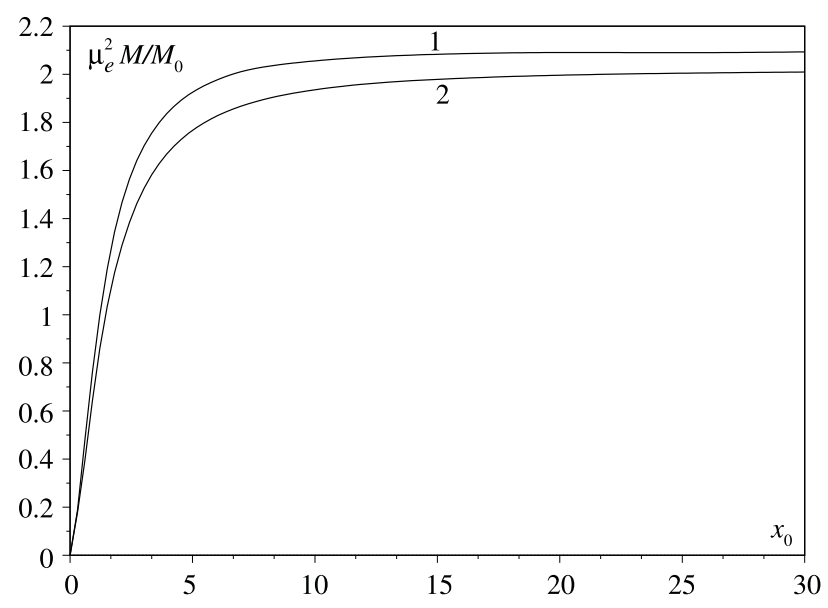

Fig. 4. Dependence of the dwarf mass $M$ on the relativistic parameter $x_{0}$ (curve 1 corresponds to the case with rotation with maximal frequency $\tilde{\Omega}_{\max }, 2$ - without rotation).

In Fig. 5 was shown dependence of the dimensionless equatorial radius of dwarf on the parameter $x_{0}$ at maximal frequency rotation (curve 1), and dwarf without rotation (curve 2). As was shown from the figure the influence of rotation is reduced to occurrence of the ellipsoidal of dwarf, and the value

$$
\left\{\xi_{1}\left(x_{0}, \pi / 2\right)-\xi_{1}\left(x_{0}\right)\right\}\left(\xi_{1}\left(x_{0}\right)\right)^{-1}
$$

is proportion to $\tilde{\Omega}^{2}$ and does not exceed $30 \%$ at maximal rotation frequency.

Let us introduce the moment of inertia of a dwarf with parameters $\mu_{e}, x_{0}, \Omega$ relative to the axis of rotation:

$$
\begin{aligned}
& I\left(x_{0}, \mu_{e}, \Omega\right)=\frac{M_{0} R_{0}^{2}}{2 \varepsilon_{0}^{2} \mu_{e}^{4}} J\left(x_{0}, \Omega\right), \\
& J\left(x_{0}, \Omega\right)=\int_{0}^{\pi} \sin ^{3} \theta d \theta \int_{0}^{\xi_{1}(\theta)} \xi^{4}\left\{y^{2}(\xi, \theta)+\frac{2}{\varepsilon_{0}} y(\xi, \theta)\right\}^{3 / 2} d \xi .
\end{aligned}
$$

As can be seen from Fig. 6, the moment of inertia depends on rotational frequency and is a nonmonotonic function of the relativistic parameter and the difference

$$
I\left(x_{0}, \mu_{e}, \Omega\right)-I\left(x_{0}, \mu_{e}, 0\right)
$$

at fixed $x_{0}$ is proportional to $\tilde{\Omega}^{2}$. 


\section{Dwarfs with spin-polarized electron subsystem}

In the work [12] it was proposed the model of a degenerate dwarf, in which electron subsystem is in the partial spin-polarization under influence of the magnetic field. In the frame of this model in the low temperature regime the momentum distribution is as follows

$$
n_{\mathbf{k}, \sigma}=\theta\left(k_{F}^{\sigma}-k\right), \quad \sigma= \pm,
$$

moreover $k_{F}^{+}>k_{F}, k_{F}^{-}<k_{F}$, where $k_{F}$ is the Fermi wave number in paramagnetic phase. The partial number density of electrons

$$
n_{+}=\frac{1}{V} \sum_{\mathbf{k}} n_{\mathbf{k},+}, \quad n_{-}=\frac{1}{V} \sum_{\mathbf{k}} n_{\mathbf{k},-}
$$

determines the degree of spin-polarization

$$
\zeta=\frac{n_{+}-n_{-}}{n_{+}+n_{-}}, \quad 0 \leqslant \zeta \leqslant 1
$$

so

$$
n_{+}=\frac{n}{2}(1+\zeta), \quad n_{-}=\frac{n}{2}(1-\zeta),
$$

where $n=n_{+}+n_{-}$, and the Fermi waves numbers for particular subsystem are following: $k_{F}^{+}=k_{F} \lambda_{+}$, $k_{F}^{-}=k_{F} \lambda_{-}, k_{F}=\left(3 \pi^{2} n\right)^{1 / 3}, \lambda_{ \pm}=(1 \pm \zeta)^{1 / 3}$. To simplify the calculations in this model the parameter $\zeta$ is considered as a constant and coordinate independent.

The expression for the pressure of an ideal polarized electron gas generalizes formula (2),

$$
\begin{aligned}
& \tilde{P}(\mathbf{r})=\sum_{\sigma= \pm} P_{\sigma}\left(x_{\sigma}(\mathbf{r})\right)=\sum_{\sigma= \pm} \frac{\pi m_{0}^{4} c^{5}}{3 h^{3}} \mathcal{F}_{\sigma}\left(x_{\sigma}(\mathbf{r})\right), \\
& \mathcal{F}_{\sigma}\left(x_{\sigma}\right)=4 \int_{0}^{x_{\sigma}} \frac{t^{4} d t}{\sqrt{1+t^{2}}}, \quad x_{\sigma}(\mathbf{r})=\hbar k_{F}^{\sigma}(\mathbf{r}) / m_{0} c=x(\mathbf{r}) \lambda_{\sigma}
\end{aligned}
$$

where $x(\mathbf{r})$ is the relativistic parameter in paramagnetic state. The ratio $\tilde{P}(\mathbf{r})$ to the pressure in paramagnetic model at $x \gg 1$ is equal $\frac{1}{2} \sum_{\sigma} \lambda_{\sigma}^{4}=1+\sum_{n \geqslant 1} a_{n} \zeta^{2 n}$ and at $x \ll 1$ the ratio is $\frac{1}{2} \sum_{\sigma} \lambda_{\sigma}^{5}=$ $1+\sum_{n \geqslant 1} b_{n} \zeta^{2 n}$, where $a_{n}, b_{n}>0$. Such increase of pressure leads to increasing mass of a dwarf.

The equilibrium equation takes the following form:

$$
\sum_{\sigma} \lambda_{\sigma}^{3} \Delta\left[\left(1+x_{\sigma}^{2}(r)\right)^{1 / 2}-1\right]=-\frac{64 \pi^{2} G\left(m_{0} c^{2} m_{u} \mu_{e}\right)^{2}}{3(h c)^{3}} x^{3}(\mathbf{r})+4 \Omega^{2} \frac{m_{u} \mu_{e}}{m_{0} c^{2}} .
$$

Proceeding to dimensionless variables $\mathbf{r}=\boldsymbol{\xi} \tilde{\lambda}$,

$$
\begin{aligned}
& y(\boldsymbol{\xi})=\left(\varepsilon_{0}^{\zeta}\right)^{-1} \sum_{\sigma} \lambda_{\sigma}^{3}\left\{\left[1+x_{\sigma}^{2}(\mathbf{r})\right]^{1 / 2}-1\right\}, \\
& \varepsilon_{0}^{\zeta}=\sum_{\sigma} \lambda_{\sigma}^{3}\left\{\left[1+x_{0}^{2} \lambda_{\sigma}^{2}\right]^{1 / 2}-1\right\},
\end{aligned}
$$

From the expression (30) we find that

$$
\begin{aligned}
& x(\mathbf{r})=2^{-1 / 2}\left(\lambda_{+}^{8}-\lambda_{-}^{8}\right)^{-1}\{b(y)-a(y)\}^{1 / 2}, \\
& a(y)=\left\{b^{2}(y)-4\left(\lambda_{+}^{8}-\lambda_{-}^{8}\right)^{2} c(y)\right\}^{1 / 2}, \\
& b(y)=2\left\{\left(\lambda_{+}^{8}+\lambda_{-}^{8}\right)\left[\left(\varepsilon_{0}^{\zeta} y\right)^{2}+4 \varepsilon_{0}^{\zeta} y\right]+4\left(\lambda_{+} \lambda_{-}\right)^{3}\left(\lambda_{+}^{5}+\lambda_{-}^{5}\right)\right\},
\end{aligned}
$$

Mathematical Modeling and Computing, Vol.4, No.1, pp. 107-115 (2017) 


$$
c(y)=\left[\left(\varepsilon_{0}^{\zeta} y\right)^{2}+4 \varepsilon_{0}^{\xi} y\right]\left\{\left[\left(\varepsilon_{0}^{\zeta} y\right)^{2}+4 \varepsilon_{0}^{\zeta} y\right]+4\left(\lambda_{+} \lambda_{-}\right)^{3}\right\} .
$$

In dimensionless form the equilibrium equation without rotation is as follows:

$$
\Delta(\boldsymbol{\xi}) y(\boldsymbol{\xi})=-\left\{\sqrt{2}\left(\lambda_{+}^{8}-\lambda_{-}^{8}\right)^{-1}\left(\varepsilon_{0}^{\zeta}\right)^{-1}[b(y)-a(y)]^{1 / 2}\right\}^{3} .
$$

Thus the length scale $\tilde{\lambda}$ is defined by condition

$$
\frac{32 \pi^{2} G}{3(h c)^{3}}\left\{m_{0} c^{2} m_{u} \mu_{e} \tilde{\lambda} \frac{\varepsilon_{0}^{\zeta}}{2}\right\}^{2}=1
$$

The equation (32) satisfies the boundary conditions $y(0)=1, y^{\prime}(0)=0$ and condition $y(\boldsymbol{\xi}) \geqslant 0$.

In work [12] the equation solutions (32) have been found using the numerical integration at fixed values of parameters $x_{0}$ and $\zeta$. Since the main object of our study are massive dwarfs, in this work we find solution of equilibrium equation of dwarf which rotation in the range of intermediate and high values of the relativistic parameter using the approximate ratio

$$
x(\mathbf{r}) \cong \varepsilon_{0}^{\zeta}\left(\lambda_{+}^{4}+\lambda_{-}^{4}\right)^{-1}\left\{y^{2}(\boldsymbol{\xi})+\frac{4}{\varepsilon_{0}^{\zeta}} y(\boldsymbol{\xi})\right\}^{1 / 2},
$$

so the equilibrium equation simplifies and takes the form of equation (12).

An important is the case of full polarization, when $\lambda_{+}=2^{1 / 3}, \lambda_{-}=0$. To compare results with ones for paramagnetic state, it is appropriate in this case to modify slightly the definition of dimensionless variables: let $\mathbf{r}=\boldsymbol{\xi} \tilde{\lambda}$,

$$
\begin{aligned}
& y_{+}(\xi, \theta)=\left(\varepsilon_{0}^{+}\right)^{-1}\left\{\left[1+\lambda_{+}^{2} x^{2}(\mathbf{r})\right]^{1 / 2}-1\right\} \\
& \varepsilon_{0}^{+}=\left[1+\lambda_{+}^{2} x_{0}^{2}\right]^{1 / 2}-1 .
\end{aligned}
$$

Then the equilibrium equation takes in the form

$$
\frac{d^{2}}{d \xi^{2}} \varphi_{+}(\xi, \theta)+\frac{2}{\xi} \frac{d}{d \xi} \varphi_{+}(\xi, \theta)=-\left\{\left[\varphi_{+}(\xi, \theta)+\omega_{+}(\xi, \theta)\right]^{2}+\frac{2}{\varepsilon_{0}^{+}}\left[\varphi_{+}(\xi, \theta)+\omega_{+}(\xi, \theta)\right]\right\}^{3 / 2},
$$

where

$$
\varphi_{+}(\xi, \theta)=y_{+}(\xi, \theta)-\omega_{+}(\xi, \theta), \quad \omega_{+}(\xi, \theta)=\tilde{\Omega}_{+}^{2} \xi^{2} \sin ^{2} \theta, \quad \tilde{\Omega}_{+}^{2} \equiv \frac{\Omega^{2} m_{u} \mu_{e} \tilde{\lambda}^{2}}{2 \varepsilon_{0}^{+} m_{0} c^{2}},
$$

and the scale $\tilde{\lambda}$ is determined by expression

$$
\frac{16 \pi^{2} G}{3(h c)^{3}}\left\{m_{0} c^{2} m_{u} \mu_{e} \tilde{\lambda} \varepsilon_{0}^{+}\right\}^{2}=1 .
$$

As can be seen from the comparison of ratios (11) and (38), the product $\tilde{\lambda} \varepsilon_{0}^{+}=\sqrt{2} \lambda \varepsilon_{0}$, therefore the expression for the mass of dwarf with full polarization subsystem of electrons can be written as

$$
\begin{aligned}
& M_{+}\left(x_{0}, \mu_{e}, \Omega\right)=\sqrt{2} \frac{M_{0}}{\mu_{e}^{2}} \mathcal{M}_{+}\left(x_{0}, \tilde{\Omega}_{+}\right) \\
& \mathcal{M}_{+}\left(x_{0}, \tilde{\Omega}_{+}\right)=\int_{0}^{\pi / 2} \sin \theta d \theta \int_{0}^{\xi_{1}^{+}(\theta)} \xi^{2}\left\{y_{+}^{2}(\xi, \theta)+\frac{2}{\varepsilon_{0}^{+}} y_{+}(\xi, \theta)\right\}^{3 / 2} d \xi
\end{aligned}
$$


where $\xi_{1}^{+}(\theta)$ is determined by the condition $y_{+}(\xi, \theta)=0$ for given values $x_{0}$ and $\tilde{\Omega}_{+}$. Hence, in the region of sufficiently large values of the parameter $x_{0}$, where $\xi_{1}(0)$ and $\xi_{1}^{+}(\theta)$ are almost independent on this parameter, mass of a dwarf with polarized electron subsystem is about in $\sqrt{2}$ times larger than the mass of dwarf with paramagnetic subsystem at the same rotation speed.

\section{Conclusions}

As was shown from the calculations, the influence of the axial rotation and spin-polarization lead to increase the mass and radius of a dwarf comparing with the standard model without rotation. Maximal increase of dwarf mass by axial rotation is about $5 \%$ in the both models. Hence, the absolute value of maximal dwarf mass with fully polarized electron system and the maximal rotation speed is about $2.15 M_{\odot}$.

[1] Adams W. S. The spectrum of the companion of Sirius. PASP. 27, 236 (1915).

[2] Fowler R. H. On dense matter. MNRAS. 87, 114 (1926).

[3] Chandrasekhar S. The maximum mass of ideal white dwarfs. Astrophys. Journ. 74, 81 (1931).

[4] Chandrasekhar S. Stellar configurations with degenerate cores' (second paper). MNRAS. 95, 676 (1935).

[5] Salpeter E. Energy and pressure of a zero-temperature plasma. Astrophys. Journ. 134, 669 (1961).

[6] Shapiro S. L. Teukolsky S. A. Black Holes, White Dwarfs and Neutron Stars. Cornell University, Ithaca, New York (1983).

[7] Hamada T., Salpeter E. Models for zero-temperature stars. Europ. Astrophys. J. 133, 683 (1961).

[8] Zeldovich Ya. B., Novikov I. D. Relativistic astrophysics. Moscow, Nauka (1967).

[9] Vavrukh M. V., Smerechinskii S. V. A finite temperature Chandrasekhar model: determining the parameters and computing the characteristics of degenerate dwarfs. Astronomy Reports. 56, n. 5, 363 (2012).

[10] Vavrukh M. V., Smerechinskii S. V. Hot degenerate dwarfs in a two-phase model. Astronomy Reports. 57, n. 2, 913 (2013).

[11] Vavrukh M., Tyshko N., Smerechynskyj S. Interparticle interactions, general relativity effects, and critical parameter of white dwarfs. Mathematical Modeling And Computing. 1, n. 2, 264 (2014).

[12] Vavrukh M. V., Dzikovskyi D. V., Tyshko N.L. Model of degenerate dwarf with spin-polarized electron system. Odessa Astronomical Publications. 28, n. 1, 82 (2015). 


\title{
Вплив осьового обертання на характеристики вироджених карликів
}

\author{
Ваврух М., Смеречинський С., Дзіковський Д. \\ Львівсъкий начіональний університет імені Івана Франка \\ вул. Кирила і Мефодія, 8, 79005 Лввів, Україна
}

\begin{abstract}
Розраховано зміну характеристик холодних вироджених карликів під впливом осьового обертання у межах три- та чотирипараметричної моделей. Показано, що відносне збільшення маси карлика становить приблизно $5 \%$. Тому максимальна маса у стандартній моделі з парамагнітною електронною підсистемою досягає $1.52 M_{\odot}$, а в моделі зі спін-поляризованою електронною підсистемою $-2.15 M_{\odot}$. Знайдено залежність форми карлика від параметрів моделей (густини у центрі зорі, параметра хімічного складу, частоти обертання і ступеня поляризації).
\end{abstract}

Ключові слова: вироджений карлик, густина у иентрі зорі, параметр хімічного складу, частота обертання, ступінь поляризацї.

2000 MSC: $85 \mathrm{~A} 15,83 \mathrm{C} 25,82 \mathrm{D} 35$

УдК: $524.31 .084,524.384,524.352 .3$ 